\title{
Der Friedensvertrag von Sèvres 1920 und die osmanische Haltung
}

\author{
The Peace Treaty of Sèvre 1920 and the Ottoman attitude
}

The Peace Treaty of Sèvre aimed to create an Armenian, Greek and Kurdish nation-state on the former soil of the Ottoman Empire. At the same time the Treaty enabled the Allies to achieve their geostrategic goals by weakening the Sublime Ports political sovereignty. This essay focusses narrowly on the content of the Treaty and how the last Islamic Dynasty struggled to achieve better political conditions to safeguard its future.

Keywords: Christian Minorities - First World War-Ottoman Empire - Treaty of Sèvre -Turkey

\section{Einleitung}

Das Ende des Ersten Weltkrieges markiert auch das Ende des Osmanischen Reiches. Auf der Seite der Mittelmächte in den Krieg eingetreten, war die Hohe Pforte nicht in der Lage, die Alliierten aufzuhalten, geschweige denn - abgesehen von Gallipoli - ihnen erhebliche Niederlagen zuzufügen. Großbritannien, Frankreich und Italien besetzten das Reich am Ende des Krieges und teilten es geografisch untereinander auf. Gleichzeitig - dies auch schon vor dem Kriegsausbruch unterstützten sie Unabhängigkeitsbestrebungen osmanischer Minderheiten, wie etwa der Armenier und Griechen. Letztere konnten vor allem mit diplomatischer Zustimmung und materieller Unterstützung der Briten das osmanische Smyrna militärisch besetzen. Die politische Förderung von ehemaligen osmanischen Minoritäten und das Vorhaben der Alliierten, einen armenischen sowie griechischen Staat auf dem Territorium der Türkei zu etablieren, ist nur bedingt dem Kontext der "self-determination“ zuzuordnen. Das ist auch die These dieses Artikels. Der Blick auf den Friedensvertrag von Sèvres verstärkt den Eindruck, dass Großbritannien durch die Schwächung der letzten muslimischen Dynastie seiner Kolonialpolitik des 19. Jahrhunderts am Ende des Ersten Weltkrieges zum Erfolg verhelfen wollte. Auch Frankreich wollte durch den Friedensvertrag seine geostrategischen Interessen im Nahen Osten sichern und dadurch die eigenen materiellen und strukturellen Herausforderungen auf französischem Boden fördern. Interessanterweise wurde das Reich der Hohen Pforte so aufgeteilt, dass der verbliebene Rest an das Territorium des Osmanischen Hauses im 7. Jahrhundert erinnerte.

Aufgrund der gebotenen Kürze dieses Artikels ist es unmöglich, den Friedensvertrag von Sèvres in ausführlicher Art und Weise im historischen Kontext kritisch zu analysieren. Deswegen liegt der Fokus dieses Beitrages auf folgenden zwei Punkten: Welche politischen Verwaltungsmöglichkeiten räumten sich die Alliierten mit Blick auf die Meerengen selbst ein? Diese Frage bot den Siegermächten - vor allem Großbritannien und Frankreich - eine Perspektive für einen $\mathrm{Zu}$ gang zum Schwarzen Meer, Nahen Osten und nach Indien. Einen weiteren geostrategisch wichtigen Vorteil bot die Kontrolle der Meerengen für die Alliierten, indem sie direkt vor der Haustüre des bolschewistischen UdSSR ihre zukünftigen 
Verbündeten positionieren konnten. Der Kommunismus wurde von den europäischen Siegermächten - auch von US-Präsident Woodrow Wilson - als eine große Gefahr für den Weltfrieden erachtet. Welche Optionen der Friedensvertrag für Armenier, Griechen und Kurden bot, wird im Anschluss sowie als zweiter Punkt in diesem Text analysiert. Auch in diesem Teil ist es nicht das Ziel, die gesamte Vorgeschichte und die Entwicklungen vor und nach dem Krieg bis zur Gründung der türkischen Republik unter Mustafa Kemal zu rekonstruieren, sondern den Vertrag anhand der einzelnen Sektionen und Artikel zu analysieren. Gleichzeitig in die Analyse des Vertrages aufgenommen ist die 34-seitige Stellungnahme der Osmanen, die in einzelnen Aussagen hier Eingang gefunden hat. Die oben erwähnten Herausforderungen erlauben es nicht, in diesem Text das Thema „Sèvres-Syndrom“ in die Untersuchung aufzunehmen. Die Leser und Leserinnen mögen dies verzeihen.

\section{Der Friedensvertrag von Sèvres und die osmanische Haltung}

Im Friedensvertrag von Sèvres vom 10. August 1920 werden die Begriffe „Türkisches Reich, Osmanisches Reich oder Türkei“ synonym verwendet. ${ }^{1}$ Gleich zu Beginn des Vertrages, in Sektion I,
Artikel 1-260, wird darauf hingewiesen, dass auf Ersuchen der kaiserlich-osmanischen Regierung der Türkei am 30. Oktober 1918 ein Waffenstillstand seitens der Alliierten gewährt worden sei. ${ }^{2}$ In diesem wurde auch festgehalten, dass im Zuge der weiteren Verhandlungen ein Friedensvertrag zwischen den Parteien geschlossen werden könnte. Am 10. August 1920 war es soweit. Zu den Unterzeichnerstaaten auf Seiten der Alliierten gehören das Britische Empire, Frankreich, Italien und Japan. Ebenso auf der Liste der Alliierten zu finden sind die „,associated Powers“3, bestehend aus Armenien, Belgien, Griechenland, dem Königreich Hedschas, Polen, Portugal, Rumänien, dem Königreich der Serben, Kroaten und Slowenen (SHS-Staat) sowie der Tschechoslowakei. Die Türkei repräsentiert sich bzw. das Osmanische Reich.

Zum ersten Mal trafen die diplomatischen Vertreter der Kriegsparteien am 17. Juni 1919 aufeinander. Bei den Pariser Friedensverhandlungen durfte eine osmanische Delegation, angeführt vom Schwager des damaligen Sultans Vahideddin, Damat Mehmet Ferit Paşa, die Interessen der Hohen Pforte vertreten. Auf der unteren Zusammenstellung sind alle Minister aufgezählt (mit ihren jeweiligen Amtsperioden), die im Rahmen der Verhandlungen des Friedensvertrags die osmanische Delegation angeführt haben:

\footnotetext{
${ }^{1}$ Vgl. ORAN, Peace Treaty of Sèvre 66.

${ }^{2}$ Der gesamte Friedensvertrag von Sèvres kann unter http://sam.baskent.edu.tr/belge/Sevres_ENG.pdf (4. 10. 2018) abgerufen werden.
}

${ }^{3}$ Ebd. 


\begin{tabular}{|c|c|c|c|}
\hline Staatsoberhaupt & Regierung & Außenminister & Generalsekretär Außenamt \\
\hline \multirow{9}{*}{$\begin{array}{l}\text { Sultan Mehmet VI. } \\
\text { [Vahideddin] } \\
\text { in Istanbul } \\
\text { 3. 7. 1918-1. 11. } 1922\end{array}$} & $\begin{array}{l}\text { Ahmet Izzet Paşa } \\
\text { [Furgaç] } \\
\text { 14. 10. 1918-11. 11. } 1918\end{array}$ & $\begin{array}{l}\text { Ahmet Nábi Bey } \\
\text { 14. 10. 1918-11. 11. } 1918\end{array}$ & \\
\hline & \multirow{2}{*}{$\begin{array}{l}\text { Ahmet Tevfik Paşa } \\
\text { [Okday] } \\
\text { 12. 10. 1918-3.3. } 1919\end{array}$} & $\begin{array}{l}\text { Mustafa Reşit Paşa } \\
\text { 12. 10. 1918-23. 2. } 1919\end{array}$ & \\
\hline & & $\begin{array}{l}\text { Yusuf Frano Paşa } \\
\text { 24. 2. 1919-3.3. } 1919 \\
\end{array}$ & \\
\hline & $\begin{array}{l}\text { Damat Mehmet Ferit Paşa } \\
\text { 4. 3. 1919-2. 10. } 1919\end{array}$ & $\begin{array}{l}\text { Damat Mehmet Ferit Paşa } \\
\text { 4. 3. 1919-2. 10. } 1919\end{array}$ & \\
\hline & \multirow[t]{2}{*}{$\begin{array}{l}\text { Ali Riza Paşa } \\
\text { 3. 10. 1919-7. 3. } 1920\end{array}$} & $\begin{array}{l}\text { Mustafa Reşit Paşa } \\
\text { 3. 10.1919-8. 2. } 1920 \\
\end{array}$ & \\
\hline & & \multirow{2}{*}{$\begin{array}{l}\text { Abdüllatif Safá Bey } \\
\text { 9. 2. 1920-4. } 4.1920\end{array}$} & \\
\hline & $\begin{array}{l}\text { Salih Hulusi Paşa } \\
\text { 8. 3. 1920-4. 4. } 1920 \\
\end{array}$ & & \\
\hline & $\begin{array}{l}\text { Damat Mehmet Ferit Paşa } \\
\text { 5. 4. 1920-17. 10. } 1920\end{array}$ & $\begin{array}{l}\text { Damat Mehmet Ferit Paşa } \\
\text { 5. 4. 1920-17. 10. } 1920\end{array}$ & \\
\hline & $\begin{array}{l}\text { Ahmet Tevfik Paşa } \\
\text { [Okday] } \\
\text { 18. 10. 1920-4. 11. } 1922\end{array}$ & $\begin{array}{l}\text { Ahmet Izzet Paşa } \\
\text { [Furgaç] } \\
\text { 14. 6.1921-4. 11. } 1922\end{array}$ & \\
\hline \multirow{10}{*}{$\begin{array}{l}\text { TGNA Präsident } \\
\text { Mustafa Kemal Paşa } \\
\text { [Atatürk] } \\
\text { in Ankara } \\
\text { 24. 4. 1920-29.10. } 1923\end{array}$} & \multirow{2}{*}{$\begin{array}{l}\text { Provisorisches Exekutiv- } \\
\text { Komitee, Präsident } \\
\text { Mustafa Kemal Paşa } \\
\text { 25.4. 1920-2. 5. } 1920 \\
\end{array}$} & & \\
\hline & & & \multirow[t]{8}{*}{$\begin{array}{l}\text { Suad Bey [Davaz] } \\
\text { 30. 4. 1920-27. } 81923\end{array}$} \\
\hline & $\begin{array}{l}\text { Erster Exekutivrat, } \\
\text { Premierminister } \\
\text { Mustafa Kemal Paşa } \\
\text { 3. 5. 1920-23. 1.1921 }\end{array}$ & $\begin{array}{l}\text { Bekir Sami Bey } \\
\text { [Kunduh] } \\
\text { 3. 5. 1920-12. 5. } 1921\end{array}$ & \\
\hline & \multirow{2}{*}{$\begin{array}{l}\text { Zweiter Exekutivrat, } \\
\text { Premierminister } \\
\text { M. Fevzy Paşa [Cakmak] } \\
\text { 24. 1. 1921-18. 5. } 1921 \\
\end{array}$} & & \\
\hline & & \multirow{3}{*}{$\begin{array}{l}\text { Yusuf Kemal Bey } \\
\text { [Tengirşenk] } \\
\text { 16. 5. 1921-25. 10. } 1922\end{array}$} & \\
\hline & $\begin{array}{l}\text { Dritter Exekutivrat, } \\
\text { Premierminister } \\
\text { M. Fevzy Paşa [Cakmak] } \\
\text { 19. 5. 1921-9.7.1922 }\end{array}$ & & \\
\hline & \multirow{2}{*}{$\begin{array}{l}\text { Vierter Exekutivrat, } \\
\text { Premierminister } \\
\text { Hüseyin Rauf Bey [Orbay] } \\
\text { 12. 7. 1922-4. 8. } 1923\end{array}$} & & \\
\hline & & $\begin{array}{l}\text { Mustafa Ismet Paşa } \\
\text { [Inönü] } \\
\text { 26. 10. 1922-22. 11. } 1924\end{array}$ & \\
\hline & \multirow{2}{*}{$\begin{array}{l}\text { Fünfter Exekutivrat, } \\
\text { Premierminister } \\
\text { Ali Fethi Bey [Okyar] } \\
\text { 14. 8. 1923-29. 10. } 1923\end{array}$} & & \\
\hline & & & $\begin{array}{l}\text { Tevfik Kemal Bey } \\
\text { [Koperler] } \\
\text { 3. 9. 1923-9. 11. } 1925\end{array}$ \\
\hline
\end{tabular}

Quelle: ORAN, Peace Treaty of Sèvre 52

Die osmanische Delegation war auf das Treffen gut vorbereitet. Ferit Paşa hielt eine furiose und diplomatisch gut ausgearbeitete Rede, die die Siegermächte dazu bewegen sollte, der Hohen 
Pforte zur alten Stärke zu verhelfen und deren Souveränität und Integrität nicht in Frage zu stellen. Dabei wurde das Argument ins Feld geführt, dass das Osmanische Reich über viele Jahrhunderte ein Garant für Sicherheit und Prosperität auf dem europäischen, arabischen und asiatischen Kontinent gewesen sei. Die Grenzen des muslimischen Reiches sollten, laut Ferit Paşa, gemäß den Grenzen von 1878 verlaufen, die Region Mosul sowie weitere Teile iranischen und russischen Territoriums an das Sultanat in Istanbul zurückgegeben werden. Wie aus verschiedenen Dokumenten des Treffens hervorgeht, waren die Alliierten von der Haltung und den Forderungen der Osmanen mehr als nur vor den Kopf gestoßen. ${ }^{4}$ Türkische Politologen und Historiker sind der Überzeugung, dass sich dadurch die angespannte Situation zwischen den ehemaligen Kriegsparteien noch mehr zum Nachteil des Osmanischen Reiches entwickelte. ${ }^{5}$ Auch Margaret MacMillan verweist in ihrem 2018 erschienenen Buch „Die Friedensmacher" darauf, dass die Ressentiments der damaligen britischen Führung gegenüber den Osmanen zu harschen Äußerungen bis hin zum Wunsch nach einer Ausmerzung des Reiches führten. ${ }^{6}$ Eine politische Aussöhnung und der Aufbau einer gemeinsamen Freundschaft war nur bedingt beabsichtigt und lediglich eine Option unter vielen.

Bevor es am 10. Mai 1920 zu einem erneuten Treffen kam, wurden seitens der Alliierten mehrere Zusammenkünfte im Vorfeld der Friedensgespräche in Sèvres abgehalten, so am 12. Februar

\footnotetext{
${ }^{4}$ Vgl. Helmreich 80-81; vgl. auch Meray, OlCAy, Osman, Osmanlı imparatorluğunun çöküş belgeleri.; ebenso KURAT, Türkiye ve Rusya.

${ }^{5}$ Vgl. ORAN, Peace Treaty of Sèvre 65.

${ }^{6}$ „Die Anwesenheit von Türken in Europa ist für jeden Betroffenen eine Quelle ungemilderten Übels gewesen. Ich kenne keine einzige Angelegenheit, ob nun türkisch oder nicht, die in 500 Jahren von dieser Angelegenheit profitiert hätte." Nicht zu Letzt deswegen, waren die Briten entschlossen, "diesen Kopf [gemeint ist die Hohe Pforte], der das Leben in Europa vergiftet", los zu werden. Vgl. MACMiLLAN, Friedensmacher 494.
}

1920 in London und später in Belgien und Spanien. In Sèvres wurde der osmanischen Delegation, diesmal angeführt von Großwesir Ahmet Tevik Paşa, ${ }^{7}$ am 11. Mai 1920 der Vertragstext ausgehändigt - ohne Informationen im Vorfeld und der anschließende Protest der türkischen Vertreter über das Vorgehen sowie die Konditionen des Friedensvertrags weitgehend ignoriert. Parallel zu den oben genannten Entwicklungen eroberten griechische Truppen in einer groß angelegten Militäroffensive Gebiete in Westanatolien sowie die Städte Balıkesir, Bursa und Uşak. ${ }^{8}$ Die osmanische Regierung in Istanbul deutete dies als ein klares Zeichen dafür, dass die Friedensverhandlungen in Sévres und die kriegerischen Ereignisse auf ihrem Territorium keine zufälligen Koinzidenzen seien und nur eines zum Ziel hätten: die weitere Schwächung, wenn nicht gar Auslöschung der Hohen Pforte. ${ }^{9}$ Diese Annahme verstärkte sich in den kommenden Wochen, als Damat Mehmet Ferit Paşa Großwesir Ahmet Tevik Paşa als Führer der Delegation ablöste und am 25. Juni 1920 in direkte Gespräche mit dem zukünftigen französischen Ministerpräsidenten Alexandre Millerand, der gleichzeitig Präsident der Friedenskonferenz in Sèvres war, eintreten wollte. Die Alliierten verweigerten aber jeden Kontakt und nach mehreren erfolglosen Versuchen verließ die osmanische Delegation am 14. Juli 1920 Sèvres Richtung Istanbul.

Sultan Mehmed VI. Vahideddin musste nun an mehreren Fronten gleichzeitig agieren, um eine politische Zukunft der Hohen Pforte zu gewährleisten, seine Macht nicht weiter zu gefährden

\footnotetext{
${ }^{7}$ Ebenso Teil der Delegation waren der Bildungsminister Fahreddin Bey und Minister für Hoch- und Tiefbau Cemil Paşa.

${ }^{8}$ Vgl. JeNSEN, The Greco-Turkish War 553-565.

${ }^{9}$ Vgl. ausführlicher dazu. MERAY, OLCAY, Osmanlı imparatorluğunun çöküş belgeleri; auch MACMiLLAN, Friedensmacher 485-503, kommt zu einer ähnlichen Einschätzung; Etwas differenzierter vgl. SMITH, Sovereignty; und ORAN, Peace Treaty of Sèvre.
} 
und weitere bewaffnete Auseinandersetzungen auf seinem Territorium einzudämmen. Der Friedensvertrag von Sèvres untergrub die Macht des Sultans sowie die Souveränität der Hohen Pforte und führte dazu, dass allein Istanbul in mindestens acht verschiedene Autoritätsbereiche aufgeteilt wurde. Der Sultan musste seine Macht mit der Meerengenkommission, der militärischen Verwaltung der Besatzungsmächte, den politischen Kommissaren Großbritanniens, Frankreichs und Italiens, der interalliierten Militärkommission für Inspektion und Organisation, der Finanzkommission, dem Verband der Schuldenkommission sowie konsularischen Gerichten teilen. ${ }^{10}$ Abgesehen davon musste die osmanische Regierung nicht nur gegen Alliierte und ihre Verbündeten aus den Reihen der ehemaligen osmanischen Minderheiten Widerstand leisten. ${ }^{11}$ Auch versprengte Militäreinheiten des Osmanischen Reiches, die unter Mustafa Kemal - später Atatürk - die Autorität des Sultans in Frage stellten, wurden bekämpft. ${ }^{12}$ Dies führte dazu, dass der Sultan und seine Berater nach der Ankunft der Delegation in Istanbul unmittelbar eine Versammlung im Yildiz-Palast einberiefen und beschlossen, den Forderungen der Alliierten sowie der "associated Powers" nachzukommen. Diese Entscheidung sollte der letzten Regierung der Hohen Pforte einen politischen Spielraum verschaffen, damit sie die gleichzeitigen bewaffneten und nichtbewaffneten Herausforderungen auf verschiedenen Ebenen erfolgreich bestreiten konnte. So unterzeichnete am 10. August 1920 eine neue osmanische Delegation unter der Führung von Hadi Paşa den Vertrag von Sèvres.

Im Zuge der Ereignisse von 1918 bis 1923 geschah das Gegenteil dessen, was der letzte osmanische

\footnotetext{
${ }^{10}$ MERAY, Olcay, Osmanlı imparatorluğunun çöküş belgeleri 43-183.

11 JENSEN, Greco-Turkish War.

${ }^{12}$ Vgl. PÖsCHL, Neutralismus zur Blockpolitik; vgl. auch GUTTSTADT, Türkei, Juden Holocaust; vgl. ebenso JÄSCHKE, Türkei als Nationalstaat 1339-1351.

${ }^{13}$ Ausführlicher zur türkischen Kulturrevolution vgl. ÇIÇEK, Martyrium. 63-88; ebenso zur Rolle des Islam
}

Herrscher mit der hohen Kunst der Diplomatie erreichen wollte. Der Sultan verlor innerhalb der türkischen Bevölkerung an Macht und Zustimmung und nach dem Sieg der Truppen von Mustafa Kemal 1923 dauerte es auch nur wenige Monate, bis die neue türkisch-republikanische Republik den Prozess der Beseitigung des osmanischen Erbe einleitete, mit dem sich die verschüttete nationale Urtradition der Türken durchsetzen konnte. ${ }^{13}$ Der Aufstieg von Mustafa Kemal und der Niedergang des letzten osmanischen Sultans hängen $u$. a. mit den Vertragsklauseln von Sèvres zusammen, die den Handlungsspielraum der Hohen Pforte beschnitten. Das war der osmanischen Regierung in Istanbul bewusst und dies versuchten ihre Vertreter in ihrem am 25. Juni 1920 überreichten 34-seitigen Bericht an die Alliierten deutlich zu machen.

Bereits in der Einleitung zur Stellungnahme kritisiert das Osmanische Reich, dass die Friedensbedingungen die Macht des Sultans zwar formell erwähnen bzw. anerkennen, sie de facto jedoch nicht vorhanden sei. So heißt es in „PART III. POLITICAL CLAUSES. SECTION I. CONSTANTINOPLE. ARTICLE 36" des Friedensvertrages explizit, dass die "rights and title“ der türkischen Regierung in Bezug auf Konstantinopel nicht durch den Vertrag beeinträchtigt würden und "His Majesty the Sultan“ weiterhin in der Stadt bleiben und diese auch verwalten/führen (maintain) dürfe. Der politische Hammerschlag durch die Alliierten erfolgte jedoch im darauffolgenden Absatz, in dem alle politischen "Zusagen" des ersten Absatzes abgemildert und die „rights and title" der Hohen Pforte nur bedingt zugesprochen werden. Vor allem der Schlusssatz ${ }^{14}$ mit der

bzw. Religion unter Atatürk vgl. ÇıçEK, Eine Politisierung 14.

${ }^{14}$ Im zweiten Teil von Art. 36 heißt es: „Nevertheless, in the event of Turkey failing to observe faithfully the provisions of the present Treaty, or of any treaties or 
Formulierung , $[$ [...] Turkey hereby agrees to accept $[\ldots]^{\prime \prime}$ macht die Zugeständnisse der Alliierten nur zu Lippenbekenntnissen und lässt erahnen, weshalb die osmanische Regierung in direkte Gespräche mit den verantwortlichen Vertragsparteien eintreten wollte. Außerdem waren die damaligen bewaffneten Auseinandersetzungen zwischen ehemals osmanischen Minderheiten und Istanbul nicht beendet und beide Kriegsparteien konnten den Ausgang noch zu ihren Gunsten beeinflussen.

Der soeben zitierte Satz und die Auswahl einer solchen "Rhetorik" sind ein wichtiges Charakteristikum des Friedensvertrages von Sèvres, dass sich wie ein roter Faden durch ebendiesen durchzieht. Ganz deutlich wird die Eingrenzung der politischen Souveränität der Hohen Pforte auch mit Blick auf die Meerengen, die durch den Vertragsabschluss de facto der Oberhoheit von Großbritannien, Frankreich und Italien unterstellt wurden. Das zeigt v. a. Artikel 178, der die Aufteilung des Marmarameeres in eine griechische (im Norden) und eine türkische (im Süden) Zone erklärt und dabei gleichzeitig festhält, dass nur die Alliierten diese Zonen für militärische Zwecke nutzen durften. ${ }^{15}$ Die Siegermächte konnten dadurch sicherstellen, dass sie auf direktem Wege nach Indien oder in den Nahen Osten gelangen konnten. Ebenso konnten sie damit Rivalen im Kampf um die erdölreichen Gegenden, wie etwa in Mossul, ausmanövrieren.

conventions supplementary thereto, particularly as regards the protection of the rights of racial, religious or linguistic minorities, the Allied Powers expressly reserve the right to modify the above provisions, and Turkey hereby agrees to accept any dispositions which may be taken in this connection."

${ }^{15}$ Vgl. "CHAPTER VIII. MAINTENANCE OF THE FREEDOM OF THE STRAITS“.

${ }^{16}$ In Art. 33 heißt es: „The Turkish Government recognises that it is necessary to take further measures to ensure the freedom of navigation provided for in Article 37 , and accordingly delegates, so far as it is concerned, to a Commission to be called the "Commission of the
In „SECTION II. STRAITS“ widmen sich die Artikel 37 bis 61 der Frage der Meerengen. Dabei heißt es, dass die Meerengen, die Dardanellen, das Marmarameer und der Bosporus in der $\mathrm{Zu}$ kunft, unabhängig von Kriegs- oder Friedenszeiten, für alle nichtmilitärischen oder militärischen Wasserfahrzeuge ohne "distinction of flag“ offen sein müssen. Nur ein Beschluss des Völkerbundes allein dürfe die Vereinbarungen des Vertrages modifizieren, verändern oder neu bestimmen. Werden die Artikel 33 und 39 aufmerksam gelesen, ${ }^{16}$ so wird die massive Eingrenzung der osmanischen Souveränität - und dies immer noch im Kontext der politischen Fragilität, die der Erste Weltkrieg erzeugt hat - deutlich sichtbar.

Die Alliierten fordern von der Hohen Pforte nicht nur bedingungslose Unterstützung für ihre strategischen Ziele, sondern auch die Garantie für die Gewährleistung sowie Realisierung der Vertragsinhalte, auch wenn dies zu ihrem Nachteil erfolgt und die politische sowie militärische Selbstbegrenzung zur Folge hat. In Artikel 39 wird explizit darauf verwiesen, dass sich der Einfluss der Meerengenkommission ebenso auf die Küste bzw. das Land erstrecken kann, falls solche Maßnahmen notwendig seien. ${ }^{17} \mathrm{Um}$ welche "execution of the provisions" es sich handeln könnte, wird nicht näher ausgeführt. Letztlich war jedoch klar, dass sich die Alliierten über die Kommission einen breiten Aktions- und Handlungsspielraum auf türkischem Boden eingeräumt hatten. Sie

Straits," and hereinafter referred to as 'the Commission," the control of the waters specified in Article 39". In Art. 39 heißt es: „The authority of the Commission will extend to all the waters between the Mediterranean mouth of the Dardanelles and the Black Sea mouth of the Bosphorus, and to the waters within three miles of each of these mouths. This authority may be exercised on shore to such extent as may be necessary for the execution of the provisions of this Section."

${ }^{17}$ Im Art. 39 heißt es: „This authority may be exercised on shore to such extent as may be necessary for the execution of the provisions of this Section." 
konnten die osmanische Regierung in Istanbul sogar zu Handlungen im alliierten Interesse drängen oder sie auch einfach übergehen. ${ }^{18}$

Vor allem in Artikel 40 wird dies von den Alliierten nochmals unterstrichen. Die vorgesehenen Mitgliedstaaten der Meerengen-Kommission werden mit unterschiedlichen Stimmrechten ausgestattet, damit sie die zukünftige Politik der Alliierten nicht torpedieren konnten: die USA - sofern sie einer eigenen Beteiligung zustimme -, das britische Empire, Frankreich, Italien, Russland - sofern sie zu einem Beitritt zum Völkerbund bereit sei -, Griechenland, Rumänien, Bulgarien und die Türkei. Hinzu kamen noch diplomatische Immunität und Privilegien, welche im Vertrag von Sèvres festgehalten werden, so dass die Kommission durch den Vertragsabschluss Befugnisse und Entscheidungen in völliger Unabhängigkeit von der "local authority“ treffen konnte. ${ }^{19}$ Nicht zuletzt deswegen hielten die Osmanen in ihrem 34-seitigen Schreiben an die Alliierten explizit fest:

"Either the Allies hold the view that Turkey should continue its existence, in which case they must treat it as a free and responsible country disposing of the means to carry out its responsibilities; or they want Turkey to disappear, in which case they will carry out the terms of the treaty themselves without expecting that the party whose defense has not been heard will sign the document or cooperate with them in its implementation. “20

Die Alliierten stärkten auch ihre Machtoptionen bezüglich der Minderheiten in der Türkei. Im Vertrag von Sèvres werden die Rechte und

\footnotetext{
${ }^{18}$ Vgl. ausführlicher dazu CRISS, Istanbul under allied occupation 70, 141.

${ }^{19}$ In Art. 42 heißt es: „The Commission will exercise the powers conferred on it by the present Treaty in complete independence of the local authority. It will have its own flag, its own budget and its separate organisation."

${ }^{20}$ Vgl. OrAN, Peace Treaty of Sèvre 66; vgl. auch MERAY; OlCAY, Osmanlı imparatorluğunun çöküş belgeleri 43-183.
}

Pflichten von Minoritäten nicht vom Völkerbund garantiert, sondern von den Alliierten selbst. Der Friedensvertrag sieht Konsultationen zwischen ersterem und letzterem vor, trotzdem waren Briten, Franzosen und Italiener weitgehend unabhängig und frei in der Umsetzung ihrer Interessens - und Minderheitenpolitik auf anatolischem Boden. ${ }^{21}$ Armenier, Griechen und Kurden wurden von den Alliierten zur Stärkung des eigenen Machtbereichs einbezogen, auch um dadurch der Hohen Pforte bzw. der Türkei weiteren politischen Handlungsspielraum zu nehmen. Betrachtet man die nationalistischen Entwicklungen und Massaker gegen Minderheiten im Osmanischen Reich vor, während und nach ${ }^{22}$ den Pariser Friedensverhandlungen, so kommt man nicht umhin, die Vorgehensweise der Alliierten als eine wenig effektive Machtdemonstration zu bezeichnen und gleichzeitig als eine ungeeignete Art der Begrenzung zukünftiger Verbrechen dieser Art. ${ }^{23}$ Ein Beispiel in diesem Zusammenhang war der Abzug großer Teile der britischen Armee am Ende des Ersten Weltkrieges bzw. im Laufe der Verhandlungen in Sèvres. Dieser führte langsam zu einer Steigerung der Gewalt zwischen den kämpfenden Kriegsparteien auf türkischem Boden und zu weiteren Grausamkeiten an Zivilisten oder Kriegsgefangenen. ${ }^{24}$

Bereits im Vorfeld der Friedensgespräche wurde ersichtlich, dass die politischen Zerwürfnisse zwischen den Alliierten über wirtschaftliche Ressourcen und Kosten des Krieges (Reparationen) keine dauerhafte Stabilität für Minderheiten gewährleisten konnten. ${ }^{25}$ Um nur ein kurzes Bei-

\footnotetext{
${ }^{21}$ Vgl. SMITH, Sovereignty 55.

${ }^{22} \mathrm{Vgl}$. KARPAT, Politicization of Islam.

${ }^{23}$ Vgl. JENSEN, Greco-Turkish War.

${ }^{24}$ Ausführlicher dazu vgl. JENSEN, Greco-Turkish War.

${ }^{25}$ Ausführlicher dazu MACMILLAN, Friedensmacher.
} 
spiel anzuführen: David Lloyd George und Georges Clemenceau begannen relativ früh, in Muodros darüber zu streiten, welche Partei die Friedensgespräche mit der Hohen Pforte führen sollte. ${ }^{26}$ Die Briten wiesen auf ihren Einsatz und ihre Verluste auf anatolischem Boden hin, die Franzosen konterten mit ihren Opfern an der Westfront. Darüber hinaus forderte Lloyd George die Bündnispartner zu einer Lösung der anstehenden Herausforderungen auf. Sie sollten die Frage nach der Deckung der zukünftigen Kosten der auf osmanischem Boden stationierten Heere beantworten. Diese Schwierigkeiten blieben aber ungelöst. Nichtsdestotrotz blieb eine mögliche Option erhalten, die die Alliierten vereinte und einen möglichen Ausweg bot: die Unterstützung der Griechen in einem Stellvertreterkrieg mit den Türken, in der Hoffnung, dass die geförderte griechische Partei am Ende den Sieg davonträgt und die Unterstützer davon profitieren können. Der griechisch-türkische Krieg wurde dann von beiden Seiten mit aller Härte geführt, am Ende konnte jedoch die Türkei den Sieg erringen. ${ }^{27}$

Im Verlauf des genannten Stellvertreterkrieges entschieden sich die Alliierten durch bilaterale Abkommen mit der Türkei, ihre zukünftigen Interessen nicht zu gefährden bzw. sie zu sichern. „As early as October 1921, the French made an agreement with the Turkish Republic, granting it de facto recognition by a Great Power. This agreement resolved the border between Cilicia and the French mandate in Syria.. ${ }^{28}$ Dies führte wiederum dazu, dass die im Friedensvertrag von Sèvres festgehaltenen Minderheitenrechte langsam, aber sicher an Gewicht verloren. Des Weiteren soll hier in gebotener Kürze aufgezeigt werden, was der Vertrag für die Armenier, Griechen und

\footnotetext{
${ }^{26}$ Vgl. Ebd. 496f.

${ }^{27}$ Ausführlicher dazu JENSEN, Greco-Turkish War.

${ }^{28}$ Vgl. auch ausführlicher dazu GüÇLÜ, Struggle for Mastery 593-603.

${ }^{29} \mathrm{Vgl}$. SMITH, Sovereignty 113-114.

${ }^{30}$ Wilson zitiert ebd. 184.
}

Kurden vorsah. Andere Minderheiten werden aus Platzgründen nicht berücksichtigt.

Die Armenier werden in der "SECTION VI" explizit erwähnt und zugleich wird festgehalten, dass der türkische Vertragspartner die Souveränität Armeniens als unabhängiger Staat akzeptiert. ${ }^{29}$ Für die Alliierten war die Errichtung einer unabhängigen und vom türkischen Staat akzeptierten armenischen Nation nicht primär eine Folge der Ereignisse von 1915 bis 1916. Wäre Letzteres der Fall, so hätte sie wahrscheinlich bereits Eingang in das Sykes-Picot-Abkommen gefunden, als die schockierenden Nachrichten der Massaker an den osmanischen Armeniern die europäische Öffentlichkeit erreichten. Vielmehr sollte die Gründung eines armenischen Staates als Schutzwall gegen die Gefahr einer kommunistischen Expansion dienen. Die Briten und Franzosen wussten um die dazu passende Haltung von US-Präsident Wilson und dessen Engagement, den Kommunismus einzudämmen. "The poison of Bolshevism was accepted readily by the world because it is protest against the way in which the world has worked", ${ }^{30}$ stellte der amerikanische Präsident fest. Der Kolonialismus und eine weltweit versäumte Politik für Minderheiten würden dem Kommunismus als Unterstützung dienen. Das Konzept der „self-determination" sollte die Völker dieser Welt zu einem friedlichen Miteinander führen. Welche Haltung die Türkei gegenüber dem Kommunismus einnehmen würde, konnte während der Verhandlungen in Sèvres nicht vorhergesagt werden. Andererseits war es der Aufmerksamkeit der Alliierten nicht entgangen, dass Mustafa Kemal in seinem Unabhängigkeitskampf von Lenin finanziert sowie materiell unterstützt worden war. ${ }^{31}$

\footnotetext{
${ }^{31}$ Vgl. ORAN, Peace Treaty of Sèvre 88-102; auch sehr empfehlenswert sind die folgenden Werke: ÖZCAN u.a., Burç, Kuşku ile komşuluk., welches in einer interdisziplinären Auseinandersetzung die Beziehungen
} 
Eine zukünftige Kooperation und politische Annäherung beider Länder konnte somit nicht ausgeschlossen werden..$^{32}$ Ein armenischer Verbündeter zwischen der Sowjetunion und der Türkei hätte den Alliierten zumindest militärische und politische Handlungsmöglichkeiten gegeben. Eine solche Strategie sollte den USA Jahrzehnte später im beginnenden Kalten Krieg durch die Einbeziehung der Türkei in den Koreakrieg und in die NATO gelingen.

Im Vertrag von Sèvres wird in Artikel 89 festgehalten, dass die „,...] Contracting Parties agree to submit to the arbitration of the President of the United States of America in question of the frontier to be fixed between Turkey and Armenia $[\ldots]^{\prime \prime}$. Dem amerikanischen Präsidenten oblag es demnach, die Grenzen zwischen verschiedenen Vertragsparteien festzulegen. Dies ist jedoch nur bedingt zutreffend, da die Siegermächte Präsident Wilson berieten, Empfehlungen abgaben und dadurch ihre Interessen gewahrt wissen wollten. Die strategisch wichtigen Städte wie Erzurum, Trabzon, Van und Bitlis wurden als armenisches Hoheitsgebiet deklariert, ebenso wurde Armenien ein Zugang zum Meer zugesichert. Um die Existenz des neu zu gründenden Staates nicht zu gefährden, sollte auch ein gewisser Teil der armenisch-türkischen Grenze entmilitarisiert werden. ${ }^{33}$ Dieses Vorhaben der Alliierten schwächte nicht nur die Macht des Sultans, sondern führte auch dazu, dass die Truppen Mustafa Kemals stetig wuchsen und an Zustimmung gewannen. Auch die weiteren im Vertrag

der beiden Länder und politischen Führer seit den Balkankriegen in den Fokus nimmt; die Monografie von HostLer, Türken und Sowjets., analysiert unter dem Aspekt des türkischen Nationalismus die politischen Interaktionen zwischen Ankara und Moskau vor und nach dem Unabhängigkeitskrieg; das Buch von BOZDAĞ, Suphi'yi kim öldürttü?, untersucht die Beziehungen zwischen Atatürk und Lenin im Kontext der Entstehung der türkisch-kommunistischen Partei, die von Mustafa Suphi gegründet worden ist.

${ }^{32}$ Vgl. ORAN, Turkish foreign policy 88-102.

${ }^{33}$ In Art. 89 der Section VI heißt es: „Turkey and Armenia as well as the other High Contracting Parties von Sèvres festgehaltenen politisch-rechtlichen Regelungen zu Gunsten der Griechen und Kurden schwächten die Autorität, Macht und das Ansehen der osmanischen Herrschaft. Parallel dazu verstärkte sich das Feindbild Armenier unter der Bevölkerung und einzelne „Rechtfertigungsversuche“ der Ereignisse von 1915 bis 1916 gewannen an Zustimmung. ${ }^{34}$

Nicht nur durch ein neu zu gründendes Armenien, sondern auch durch die Gründung einer kurdischen Autonomieregion mit einem zeitlich unbestimmten Hinweis auf die Möglichkeit der Schaffung eines Kurdenstaates ${ }^{35}$ wollten die Alliierten die Schwächung des Sultans vorantreiben. Die im Vertrag festgehaltenen Punkte sollten von einer Kommission - bestehend aus Briten, Franzosen und Italienern - realisiert werden. In Artikel 62 der SECTION III heißt es, dass die Alliierten sechs Monate nach Inkrafttreten des Vertrages „will prepare a scheme autonomy for the predominantly Kurdish areas“. Gemeint waren die Regionen südlich des Euphrats entlang der südlichen Grenze von Armenien sowie der nördlichen türkisch-syrischen Grenze. Entlang dieser Linien konnten die Siegermächte ihre geostrategischen Vorteile weiter ausbauen und somit ihren Zugang nach Indien oder in den Nahen Osten sichern und - wie bereits festgehalten - möglichen Rivalen wichtige Wege in warme Gewässer abschneiden.

Die Vertragsinhalte von Sèvres führten dazu, dass die Kurden den Unabhängigkeitskrieg von Mustafa Kemal unterstützten. Letzteres geschah

agree to submit to the arbitration of the President of the United States of America the question of the frontier to be fixed between Turkey and Armenia in the vilayets of Erzerum, Trebizond, Van and Bitlis, and to accept his decision thereupon, as well as any stipulations he may prescribe as to access for Armenia to the sea, and as to the demilitarisation of any portion of Turkish territory adjacent to the said frontier."

${ }^{34} \mathrm{Vgl}$. dazu die ausgezeichnete Analyse von KURAT, Türkiye ve Rusya, in den Kapiteln IV, V und VI.

${ }^{35}$ Dazu vgl. Art. 64. 
vor allem, nachdem die Alliierten die Herrschaftsverhältnisse unter den kurdischen Gruppen in der Türkei falsch eingeschätzt hatten und ihnen auch keinen direkten Weg zur Souveränität boten. Artikel 64 zeugt von dieser Unwissenheit. Die osmanischen Kurden sollten in Absprachen, Plebisziten und Verhandlungen mit dem Völkerbund diesen davon überzeugen, dass die Mehrheit der Kurden einen unabhängigen Staat bevorzuge. Aus einer politischen Perspektive war damit die Macht der kurdischen Klanführer untergraben. Staatliche Institutionen und das Vertrauen in sie, so wie in Großbritannien, Frankreich oder den USA, hatten in der Region der Kurden noch keine Tradition. Und so sahen die damaligen kurdischen Autoritäten ihre Macht durch die Aussicht auf ein Plebiszit nicht bestätigt, sondern vielmehr in Gefahr. Hier zeigte sich auch das Geschick von Mustafa Kemal, der sich nur bedingt $\mathrm{zu}$ traditioneller Herrschaftsordnung auf dem Boden der Hohen Pforte äußerte und erst nach Festigung seiner Macht grundlegende Veränderungen an der politischen Ordnung und Kultur vornahm. ${ }^{36}$

Abgesehen von den Vertragsinhalten sorgte der griechisch-türkische Krieg dafür, dass die Kurden sich auf die Seite ihrer "Glaubensbrüder" stellten. Inwieweit dabei die Religion eine Rolle gespielt hat, kann heute nicht adäquat nachgewiesen werden. ${ }^{37}$ Vielmehr war es die Uneinigkeit der Alliierten, die im Zeitraum von 1920 bis 1923 eine gemeinsame Linie nicht aufrechthalten konnten, welche dafür sorgte, dass Minoritäten auf deren Unterstützung verzichteten. Gerade die griechischen Ambitionen auf dem Boden der Hohen Pforte und die unterschiedlichen Interessen der Briten, Franzosen und Italiener hatten

\footnotetext{
${ }^{36}$ Vgl. dazu ausführlicher ÇIÇEK, Martyrium; letzter Artikel nimmt unter dem Aspekt des politischen Martyriums den türkischen Unabhängigkeitskrieg sowie Kult um Mustafa Kemal Atatürk in den Fokus.

${ }^{37}$ Die türkischen Diskussionen zu diesem Thema können hier nicht wiedergegeben werden. Stellvertretend soll hier auf das Werk von ŞENERMEN, Atatürk, hingewiesen werden.
}

ebenso Einfluss auf den Erfolg des türkischen Unabhängigkeitskrieges. Während Lloyd George die Griechen als wichtige Verbündete für die $\mathrm{Zu}$ kunft ausmachte, teilten die Franzosen und Italiener nur bedingt diese Vision. ${ }^{38}$ London hatte mit dem für sich erfolgreichen Ende des Ersten Weltkrieges die Chance, die eigenen Kolonien in Indien über das ehemalige Territorium der Hohen Pforte zu kontrollieren, ohne auf die mühsame Bündnispolitik mit Istanbul angewiesen zu sein. Ganz im Gegenteil, Großbritannien konnte nun den ehemaligen Verbündeten und gegenwärtigen Feind maßgeblich zur Akzeptanz seiner Interessen zwingen. Clemenceau wollte dagegen vor allem eine starke Achse zwischen Paris, London und Washington, um auf europäischem Boden die deutsche Gefahr zu bannen. ${ }^{39}$ Im Nahen Osten, etwa in Syrien, waren die Rivalitäten zwischen den Alliierten am politischen Horizont mehr als nur sichtbar. Auch der ambitionierte Friedensvertrag von Sèvres konnte daran nichts ändern. Mehrere Artikel ${ }^{40}$ der ,SECTION IV. SMYRNA“ haben zum Ziel, die Interessen der osmanischen Griechen zu stärken und ihnen mehr Territorium zur Verfügung zu stellen. Die gesamte Provinz von Izmir und ein Drittel des westlichen Teils der Provinz Manisa wurde mit Artikel 66 de facto zum griechischen Hoheitsgebiet. Das Ziel der Alliierten war es, die Gründung einer triangulären Front, bestehend aus Armeniern, Griechen und Kurden, auf anatolischem Boden sicherzustellen. Thre große Herausforderung bestand nicht nur darin, die einzelnen Gruppen davon zu überzeugen, ihr Recht auf Selbstbestimmung für sich zu beanspruchen, sondern auch im alliierten Lager selbst für Einigkeit zu sorgen. Der

\footnotetext{
${ }^{38}$ Helmreich, From Paris to Sèvres 100; ebenso auch die Ausführungen von SMITH, Sovereignty.

${ }^{39}$ MacMillan, Friedensmacher 217-281.

${ }^{40}$ Art. 66, 68, 69, 71, 72, 76 und 79. Auch die Art. 140 bis 151 sollten den Einfluss und die Macht der ehemaligen osmanischen Minderheiten stärken.
} 
gemeinsame Feind, die Hohe Pforte, konnte jedoch nur bedingt und für eine gewisse Zeit die Siegermächte zu einem Streben nach politischem Einklang führen.

\section{Schluss}

Ein wichtiger Grund für das Scheitern des Friedensvertrags von Sèvres war das Vorgehen der Alliierten und ihre politischen Zerwürfnisse. Ebenso führte auch die passive Rolle der USA dazu, dass sich auf dem Boden der bis dahin regierenden Hohen Pforte der Vertrag und dessen Inhalte nicht durchsetzen konnten. Die Einrichtung internationaler Kommissionen im Rahmen des Friedensvertrages - jedoch immer mit Beteiligung von Großbritannien, Frankreich und Italien - war ein politisch geschicktes Instrument zur Sicherung der eigenen geopolitischen Interessen. Wie am Beispiel der Meerengen kompakt dargestellt, diente die Aufteilung Istanbuls in acht verschiedene Autoritäts- bzw. Verwaltungszonen dazu, die Macht des Sultans einzuschränken und eigene geostrategische Ziele besser verfolgen zu können.

Die Uneinigkeit der Siegermächte führte dazu, dass Großbritannien unter anderem seine Streitkräfte frühzeitig aus Anatolien abzog und somit die Durchsetzung der Vertragsinhalte nicht realisiert werden konnte. Außerdem führten diese Differenzen wiederum dazu, dass die im Vertrag für armenische, griechische, kurdische sowie andere Minoritäten vorgesehenen Territorien nicht geschaffen und entsprechende Minderheitenrechte auch nicht durchgesetzt werden konnten. Im Falle der Kurden hatte dies sogar die Unterstützung des türkischen Unabhängigkeitskrieges Mustafa Kemals zur Folge. So lässt sich zusammengefasst konstatieren, dass die im Friedensvertrag von Sèvres festgehaltenen Minderheitenrechte und das Ziel der „self-determination “ eine politische Totgeburt waren.

\section{Korrespondenz:}

Dr. Hüseyin I. ÇIÇEK

Institut für Islamisch-Theologische Studien

Schenkenstraße 8-10

1010 Wien

hueseyin.cicek@univie.ac.at

ORCID-Nr. 0000-0003-1716-8806

\section{Abkürzungen:}

TGNA Turkish Grand National Assembly Siehe auch das allgemeine Abkürzungsverzeichnis: [http://www.rechtsgeschichte.at/files/abk.pdf]

\section{Literatur:}

İsmet Mustafa BOzDAĞ, Suphi'yi kim öldürttü? Atatürk mü, Lenin mi? (= Emre Yayınları / Yakın tarih serisi 5) (İstanbul 1992).

Hüseyin I ÇıçEK, Martyrium - Opferstatus - Gewalt, in: Zeitschrift für Genozidforschung $10 \mathrm{Heft} 2$ (2009) 63-88.

DERS., Eine Politisierung der Religion von oben, in: Die Furche 77 Nr. 13 (2017) 15.

Nur B CRISS, Istanbul under allied occupation, 19181923 (= The Ottoman Empire and its heritage 17, Leiden 1999).

Corry GutTSTADT, Die Türkei, die Juden und der Holocaust (Berlin-Hamburg 2008).

Yücel GÜÇLÜ, The Struggle for Mastery in Cilicia: Turkey, France, and the Ankara Agreement of 1921, in: International History Review 23 (2001) 593-603.

Charles W. HostLER, Türken und Sowjets. Die historische Lage und die politische Bedeutung der Türken und der Türkvölker in der heutigen Welt (Berlin 1960).

Paul C. HelmReich, From Paris to Sèvres. The partition of the Ottoman Empire at the Peace Conference of 1919-1920 (Columbus 1974).

Gotthard JÄsCHKE, Die Türkei als Nationalstaat seit der Revolution Mustafa Kemals (Atatürk)1920-1974, in: Theodor SCHIEDER (Hg.), Europa im Wandel von der Antike zum Mittelalter (7/2) (Stuttgart 1979) 1339-1351.

Peter K JenSEN, The Greco-Turkish War, 1920-1922, in: International Journal of Middle East Studies 10 (1979) 553-565.

Kemal H KARPAT, The politicization of Islam. Reconstructing identity, state, faith, and community in 
the late Ottoman state (= Studies in Middle Eastern history, Oxford u. a. 2001).

Akdes N.KURAT, , Türkiye ve Rusya. XVIII. yüzyil sonundan Kurtuluş Savaşina kadar Türk-Rus ilişkileri (1798-1919) (Ankara 2011).

Margaret MACMILLAN, Die Friedensmacher. Wie der Versailler Vertrag die Welt veränderte (Berlin 2018).

Seha Meray, Osman Olcay, Osmanlı imparatorluğunun çöküş belgeleri. Mondros bırakışması, Sevr andlaşması, ilgili belgeler (= Ankara Üniversitesi - Siyasal Bilgiler Fakültesi 409, Ankara 1977).

Baskın ORAN, The Peace Treaty of Sèvre, in: ORAN, (Hg.), Turkish foreign policy, 1919-2006. Facts and analyses with documents (= Utah series and Turkish and Islamic studies, Salt Lake City 2010) 6377.

DERs. u.a. (Hg.), Burç, Kuşku ile komşuluk. Türkiye ve Rusya ilişkilerinde değişen dinamikler (= İletişim yayınları Bugünün kitapları 219, İstanbul 2017)

Rainer PösCHL, Vom Neutralismus zur Blockpolitik. Hintergründe der Wende in der türkischen Außenpolitik nach Kemal Atatürk (München 1985).

Sedat ŞENERMEN, Atatürk, İslâm ve laiklik cumhuriyet dönemi din öğretimi ve eğitimi (İstanbul 2015).

Leonard V SMITH, Sovereignty at the Paris Peace Conference of 1919 (= The greater war 1912-1923, Oxford-New York 2018). 